\title{
Vacuum polarization energy of a complex scalar field in a vortex background
}

\author{
N. Graham $\oplus^{1}$ and H. Weigel $\oplus^{2}$ \\ ${ }^{1}$ Department of Physics, Middlebury College Middlebury, Vermont 05753, USA \\ ${ }^{2}$ Institute for Theoretical Physics, Physics Department, Stellenbosch University, \\ Matieland 7602, South Africa
}

(Received 4 February 2020; accepted 17 March 2020; published 7 April 2020)

\begin{abstract}
Scattering methods make it possible to compute the effects of renormalized quantum fluctuations on classical field configurations. As a classic example of a topologically nontrivial classical solution, the Abrikosov-Nielsen-Olesen vortex in U(1) Higgs-gauge theory provides an ideal case in which to apply these methods. While physically measurable gauge-invariant quantities are always well behaved, the topological properties of this solution give rise to singularities in gauge-variant quantities used in the scattering problem. In this paper we show how modifications of the standard scattering approach are necessary to maintain gauge invariance within a tractable calculation. We apply this technique to the vortex energy calculation in a simplified model, and show that to obtain accurate results requires an unexpectedly extensive numerical calculation, beyond what has been used in previous work.
\end{abstract}

DOI: 10.1103/PhysRevD.101.076006

\section{INTRODUCTION}

Topological vortex configurations appear in many field theory models in the form of axially symmetric $\mathrm{U}(1)$ vector potentials pointing in azimuthal direction. Although physically measurable quantities remain well defined, in these models the field profiles, which depend on the gauge choice, necessarily have a singular structure. This structure hampers many computations that go beyond the (static) mean-field approach, particularly the quantum correction to the classical static energy density per unit length. At oneloop order this quantity is given by the vacuum polarization energy (VPE), the renormalized shift in the sum of the zero point energies of the quantum fluctuations. Calculations of the VPE often use auxiliary quantities like Feynman diagrams and/or expansion schemes for scattering data [1]. These quantities are not necessarily gauge invariant and thus the singular structure matters. Throughout this paper we use the term singular for the gauge field configuration, keeping in mind that the magnetic field describing the vortex is nonsingular.

The classic example of a topological string solution is the Abrikosov-Nielsen-Olesen (ANO) vortex [2,3]. In this paper we use a simplified model that contains all the relevant subtleties and obstacles of the full Nielsen-Olesen

Published by the American Physical Society under the terms of the Creative Commons Attribution 4.0 International license. Further distribution of this work must maintain attribution to the author(s) and the published article's title, journal citation, and DOI. Funded by SCOAP ${ }^{3}$. model, but is computationally simpler. As in the full model, we consider a complex scalar field (representing a Higgs boson in particle physics or a Cooper pair condensate in condensed matter physics) coupled to the U(1) gauge field of electromagnetism. However, we consider only fluctuations of the scalar field; adding the gauge field fluctuations requires a calculation that numerically is significantly more costly, but will follow the same formalism [4].

The quantum fluctuations propagate in a combined potential generated by both the gauge and scalar field backgrounds. Typically the latter provides the dominant contribution, but the correct identification and renormalization of the ultraviolet divergences requires a detailed analysis of the interaction with the vortex background.

Formally, calculating the leading quantum correction to any classical quantity requires computing the functional determinant of the quantum fluctuations in the background of the classical configuration. To our knowledge the only full calculation of the renormalized quantum correction in the full Nielsen-Olesen model, as is made possible by the approach we will describe, is that of Refs. [5,6]. While Ref. [5] estimates the instanton transition rate, Ref. [6] indeed focuses on the quantum correction to the classical energy (per unit length of the vortex). The wave equations solved in these two papers are similar to those we will integrate. However, our approach will differ in several interconnected ways from Ref. [6]: We carry out the calculation directly for the total energy, rather than as an integral over the energy density; we use analytic continuation to carry out the integral over the wave number $k$ on the imaginary axis; and, most importantly, we express the 
calculation in a way that avoids superficial divergences originating from the singular gauge field profile. This process eliminates the need for the arbitrary additional interaction vertex that was added (and, correspondingly, subtracted back out) in Ref. [6]. We will see that formidable numerical challenges remain even after these simplifications, which suggests that the previous results are numerically unreliable within a realistic computational budget. Our analytic continuation approach also sheds light on a puzzle first exhibited in Ref. [7], which showed that calculations of quantum effects in a vortex background appear artificially convergent when computed on the real $k$ axis; by carrying out the computation in the complex $k$ plane from the outset, we will see how the appropriate divergences emerge from the analytic structure of the scattering data associated with the quantum fluctuations.

The complications that our method is capable of addressing can be particularly acute in heat kernel methods, which are typically combined with $\zeta$-function regularization, that have also been applied to vortex systems [8-10]. Not only do these methods involve complicated recursion relations for the heat kernel coefficients that need to be truncated, but the $\zeta$-function regularization also makes the implementation of standard, perturbative renormalization conditions difficult up to the point that gauge variant counterterms are required [8]. For certain geometries, however, these methods have analytic solutions [11].

This paper is structured in eight short sections. Following this introduction, we introduce the model in Sec. II and collect the relevant ingredients to compute the VPE using spectral methods for configurations that are translationally invariant in (at least) one spatial variable in Sec. III. In Sec. IV we apply this method to regular configurations that couple to a (complex) scalar quantum field. In Sec. V we show how the ultraviolet divergences of Feynman diagrams relate to the asymptotic behavior of the scattering data that enter the VPE calculation. This analysis ensures the correct identification of the ultraviolet divergences on the scattering side of the calculation. We also discuss obstacles to computing the Feynman diagrams when the background configuration is singular. Section VI gives the main analytic calculation: We show how to modify the standard approach for singular configurations for which the ingredients of the standard approach are not well defined. In Sec. VII we perform numerical experiments for this modification and demonstrate that the numerical simulation requires a sophisticated computation to match the asymptotic behavior of the scattering data as given by the analysis of the Feynman diagrams. We consider Feynman diagrams and scattering data in parallel because (i) both represent or can be expanded to represent the contribution to the VPE of a specific order of the potential generated by the vortex and (ii) Feynman diagrams provide important consistency checks on the large momentum behavior of scattering data. Finally, in Sec. VIII we summarize our findings and provide an outlook on how they affect the VPE calculation in the full model that also includes gauge field loops.

\section{THE MODEL}

In rescaled variables, the Lagrangian for this model reads

$$
\mathcal{L}=\left|\left(\partial_{\mu}-\mathrm{i} A_{\mu}\right) \phi\right|^{2}-\frac{m^{2}}{4}\left(|\phi|^{2}-1\right)^{2} .
$$

The background vortex is translationally invariant along an axis that we choose to be the $z$ axis. In terms of polar coordinates $\rho$ and $\varphi$ in the perpendicular plane, the vortex is characterized by two radial functions:

$$
\phi_{0}=f_{H}(\rho) \mathrm{e}^{\mathrm{i} \xi \varphi} \quad \text { and } \quad \boldsymbol{A}=-\hat{\boldsymbol{\varphi}} \frac{f_{G}(\rho)}{\rho} .
$$

The ANO string $[2,3,12]$ emerges as the stationary solution when $\mathcal{L}$ is supplemented by the gauge kinetic term $-(1 / 4)\left(\partial_{\mu} A_{\nu}-\partial_{\nu} A_{\mu}\right)^{2}$. The boundary conditions for the classical scalar field in the vortex configuration are always given by $\lim _{\rho \rightarrow 0} f_{H}(\rho)=0$ and $\lim _{\rho \rightarrow \infty} f_{H}(\rho)=1$. A nontrivial topology requires $\lim _{\rho \rightarrow 0} f_{G}(\rho)-\lim _{\rho \rightarrow \infty} f_{G}(\rho) \neq 0$, but the specific values depend on the choice of gauge. That choice affects the scalar field via $\xi=1-\lim _{\rho \rightarrow 0} f_{G}(\rho)$. Calculating the VPE will require the Fourier transforms of the classical vortex field configurations. In particular for the vector potential we have

$\tilde{\boldsymbol{A}}(\boldsymbol{p})=\int d^{2} x \mathrm{e}^{\mathrm{i} \mathbf{p} \cdot \mathbf{x}} \boldsymbol{A}(\boldsymbol{x})=2 \pi \mathrm{i} \hat{\boldsymbol{\varphi}}_{p} \int_{0}^{\infty} d \rho f_{G}(\rho) J_{1}(\rho p)$,

where $\hat{\boldsymbol{\varphi}}_{p}$ is the azimuthal unit vector in momentum space and $J_{1}(z)$ is a Bessel function. The integral on the righthand side is well defined only when $\lim _{\rho \rightarrow \infty} f_{G}(\rho)=0$. Hence computing the VPE requires us to choose the socalled singular gauge with $\lim _{\rho \rightarrow \infty} f_{G}(\rho)=0$, meaning that $\lim _{\rho \rightarrow 0} f_{G}(\rho) \neq 0$ for a configuration with nontrivial topology. In Sec. V we will actually see that even with this particular condition on the gauge profile, special care is needed when using Eq. (3) to compute Feynman diagrams.

We introduce small amplitude fluctuations about the classical scalar field via $\phi=\phi_{0}+\eta$ and derive their field equations in the harmonic approximation,

$$
\begin{aligned}
& {\left[\partial_{t}^{2}-\nabla^{2}+\frac{2 \mathrm{i}}{\rho^{2}} f_{G}(\rho) \partial_{\varphi}+\frac{1}{\rho^{2}} f_{G}^{2}(\rho)-\frac{3 m^{2}}{2} f_{H}^{2}(\rho)+\frac{m^{2}}{2}\right] \eta} \\
& \quad=0 .
\end{aligned}
$$

The partial wave-decomposition $\eta=\mathrm{e}^{-\mathrm{i} \omega t} \sum_{\ell} \eta_{\ell}(\rho) \mathrm{e}^{\mathrm{i} \ell \varphi}$ yields 


$$
\begin{aligned}
& {\left[-k^{2}-\frac{1}{\rho} \partial_{\rho} \rho \partial_{\rho}+\frac{\ell^{2}}{\rho^{2}}-\frac{2 \ell}{\rho^{2}} f_{G}(\rho)+\frac{1}{\rho^{2}} f_{G}^{2}(\rho)+V_{H}(\rho)\right]} \\
& \quad \times \eta_{\ell}(\rho)=0,
\end{aligned}
$$

with the dispersion relation $\omega^{2}=k^{2}+m^{2}$ and the abbreviation $V_{H}(\rho)=\frac{3 m^{2}}{2}\left(f_{H}^{2}(\rho)-1\right)$ for the scalar potential. We see that the singular gauge boundary conditions described above turn Eq. (5) into a free radial differential equation at spatial infinity. As a result, we can see explicitly that the Green's function constructed from $\eta_{\ell}$ will asymptotically approach its noninteracting counterpart so that the vacuum energy density associated with the fluctuations vanishes at spatial infinity. This is another reason for choosing the singular gauge. Then the singular behavior as $\rho \rightarrow 0$ is unavoidable meaning that the textbook [13] proof of analyticity of the scattering data cannot be applied. We will develop a procedure to remedy that obstacle.

\section{SCATTERING DATA AND VACUUM POLARIZATION ENERGY}

Equation (5) defines a potential scattering problem for which we can obtain phase shifts and bound state energies in each partial wave. Spectral methods use these scattering data to compute the VPE [1]. In this approach, we first write the vacuum expectation value of the energy density operator in the presence of the background fields in terms of the Green's function, which we can then write as a sum over the full set of solutions to Eq. (5). After subtracting the analogous contribution without the background and integrating over space, we obtain an expression for the total energy in terms of an integral over the wave number $k$ and a sum over partial waves $\ell$. In this expression, the essential element, which encodes the dynamical information, is the momentum derivative of the logarithm of the Jost function. For real momenta, this result can be expressed in terms of the phase of the Jost function, i.e., the phase shift [14]. For numerical purposes, this momentum derivative is typically treated via integrating by parts. Our calculation will take advantage of the freedom to add an arbitrary constant to this logarithm without changing the VPE in order to maintain its analytic properties. In particular, we will show that by introducing a uniquely determined constant, which does not affect the calculation on the real $k$ axis, we are able to calculate the integral by continuing to imaginary wave number $t=\mathrm{i} k$. There are then two ingredients to the integral. One arises from the discontinuity of $\ln \left(m^{2}-t^{2}\right)$ and the other from the poles of the logarithmic derivative. These poles emerge at the bound state wave numbers, thereby canceling the bound state contribution to the VPE [15]. Furthermore, on the imaginary axis functions that oscillate for real momenta [7] are replaced by their exponentially damped counterparts, making the calculation numerically more efficient. Finally, as we will see below, it is on the imaginary axis that we are able to recognize the full divergence structure of the theory.

The interface formalism [16] provides an extension of the scattering approach to compute the VPE in cases where the background potential is translationally invariant in one or more spatial coordinates. To implement this formalism in our Higgs-gauge vortex model, we first define

$$
[\nu(t)]_{n}=\lim _{L \rightarrow \infty} \sum_{\ell=-L}^{L}\left[\nu_{\ell}(t)\right]_{n},
$$

where $\nu_{\ell}(t)$ is the logarithm of the Jost function associated with orbital angular momentum $\ell$. The subscript $n$ denotes a suitable subtraction, typically in the form of the Born approximation, such that the integral in

$$
E_{\mathrm{VPE}}=\frac{1}{2 \pi} \int_{m}^{\infty} t d t[\nu(t)]_{n}+E_{\mathrm{FD}}^{(n)}+E_{\mathrm{CT}}
$$

is finite. The lower limit of the integral is the mass of the quantum fluctuations, which for the current conventions is the scalar mass $m$, and the integral runs along the branch cut discontinuity mentioned above. The subtractions in Eqs. (6) and (7) are added back as (dimensionally) regularized Feynman diagrams $E_{\mathrm{FD}}^{(n)}$; when combined with the counterterm contributions $\left(E_{\mathrm{CT}}\right)$, the regulator may be removed in a renormalizable theory. In the current study we are primarily interested in the structure of $[\nu(t)]_{n}$, in particular with regard to a singular vortex background. Therefore we leave the details of $E_{\mathrm{FD}}^{(n)}$ and $E_{\mathrm{CT}}$ with on-shell renormalization conditions [17] to the forthcoming manuscript [4], which computes the VPE in the complete Nielsen-Olesen model.

To numerically compute $\nu_{\ell}(t)$, we first write $\eta_{\ell}(\rho)=$ $K_{|\ell|}(t \rho) \bar{\eta}_{\ell}(\rho)$, where $K_{\ell}$ is a modified Bessel function. This produces the differential equation

$$
\frac{1}{\rho} \partial_{\rho} \rho \partial_{\rho} \bar{\eta}_{\ell}=2 t Z_{\ell}(t \rho) \partial_{\rho} \bar{\eta}_{\ell}+\frac{1}{\rho^{2}}\left[f_{G}^{2}-2 \ell f_{G}\right] \bar{\eta}_{\ell}+V_{H} \bar{\eta}_{\ell}
$$

with $Z_{\ell}(z)=\frac{K_{|\ell|+1}(z)}{K_{|\ell|}(z)}-\frac{|\ell|}{z}$.

We then integrate this differential equation with the boundary conditions $\lim _{\rho \rightarrow \infty} \bar{\eta}_{\ell}(\rho)=1$ and $\lim _{\rho \rightarrow \infty} \frac{d}{d \rho} \bar{\eta}_{\ell}(\rho)=0$, and extract $\nu_{\ell}(t)=\lim _{\rho \rightarrow 0} \ln \left(\bar{\eta}_{\ell}(\rho)\right)$. The Born series, which is the standard tool to remove the divergences in Eqs. (6) and (7) [1], expands the solutions of Eq. (8) in powers of the strength of the background potential $V_{B}=\frac{1}{\rho^{2}}\left[f_{G}^{2}-2 \ell f_{G}\right]+V_{H}$. This expansion produces a series that approximates the scattering data well at large momenta. Hence subtracting the leading terms of this series from $\nu(t)$ renders the integral in Eq. (7) finite. 


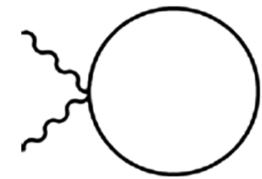

(a)

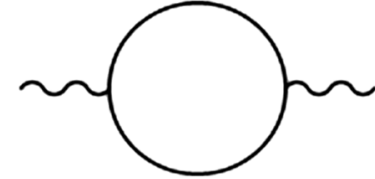

(b)

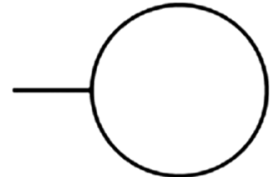

(c)

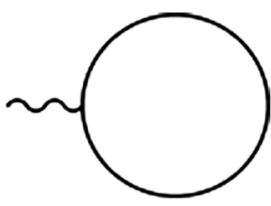

(d)

FIG. 1. Quadratically divergent one-loop diagrams.

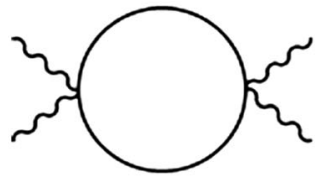

(a)

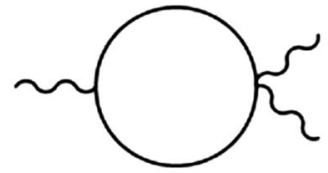

(b)

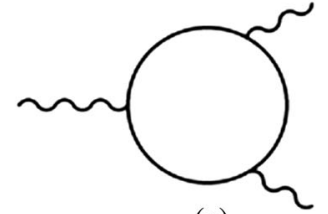

(c)

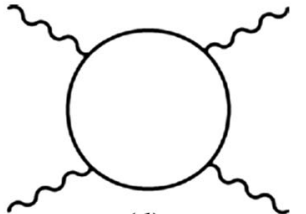

(d)

FIG. 2. Logarithmically divergent one-loop diagrams with external photon lines only.

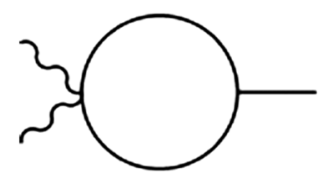

(a)

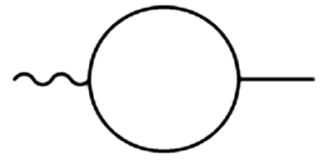

(b)

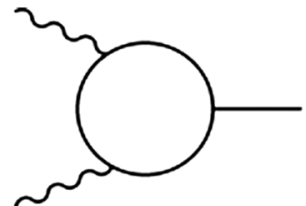

(c)

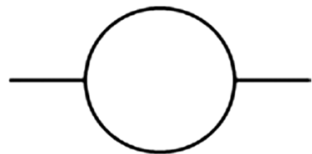

(d)

FIG. 3. Logarithmically divergent one-loop diagrams with at least one insertion of the Higgs potential $V_{H}=\frac{3 m^{2}}{2}\left(f_{H}^{2}-1\right)$.

In contrast to the phase shift, which is the imaginary part of $\nu_{\ell}$ for real momenta, the Jost function on the imaginary momentum axis is not a gauge invariant quantity. Hence we cannot rely on gauge invariance when investigating the divergence structure associated with $\nu_{\ell}$.

\section{STANDARD TREATMENT FOR REGULAR VECTOR POTENTIALS}

Before addressing singular configurations, we review the standard treatment for a regular gauge profile that vanishes at the origin and spatial infinity, in order to properly identify the quantum field theory divergences in Eq. (7). Because there are cancellations of divergences between various contributions at different orders of $V_{B}$, it is appropriate to consider insertions of $f_{G}^{2} / \rho^{2},-2 \ell f_{G} / \rho^{2}$ and $V_{H}$ separately. The first two of these terms originate from the $A_{\mu} A^{\mu}$ and $A_{\mu} \partial^{\mu}$ interactions, respectively, in Eq. (1) and correspond to vertices with one or two external photon lines, while the third corresponds to vertices with one or two external scalar lines. The full VPE can then be written as the sum of one-loop Feynman diagrams with (the Fourier transforms of) the profiles emerging as external lines, combined with the corresponding contributions of renormalization counterterms to the low-order diagrams to

\footnotetext{
${ }^{1}$ Note that we work in a gauge where the vortex profile obeys $\partial_{\mu} A^{\mu}=0$.
}

form a convergent result. In our simplified model, only the complex scalar field is quantized and appears within loops in the diagrams.

In the covariant formulation, Feynman diagrams are generated by expanding the effective action for a complex scalar field

$$
\mathcal{A}_{\mathrm{eff}}=\mathrm{i} \operatorname{Tr} \log \left[\partial^{2}+m^{2}+V\right]
$$

with respect to the components of

$$
V=\frac{3 m^{2}}{2}\left(\left|\phi_{0}\right|^{2}-1\right)-A_{\mu} A^{\mu}+\mathrm{i}\left(A^{\mu} \vec{\partial}_{\mu}+\overleftarrow{\partial}_{\mu} A^{\mu}\right),
$$

where the arrow indicates the direction of differentiation to be applied in the functional trace, Eq. (9). Here we have written the effective action for a general interaction. For the vortex configuration we will replace $V$ by $V_{B}$ defined above.

In Figs. 1-3 we display all one-loop diagrams that are divergent by power counting. There are considerable simplifications arising from relationships between the divergent parts of different diagrams, but not all of them can be straightforwardly implemented in Eq. (7). First we note that the divergences from diagrams with an odd number of gauge fields, Figs. 1(d), 2(b), 2(c), and 3(b) will have coefficients that are spatial integrals involving $\partial_{\mu} A^{\mu}=0$ for the vortex, which will vanish. Hence we 
do not need to consider these diagrams any further. Actually diagrams 1(d) and 2(b) are identically zero for the vortex.

Figure 1 shows the Feynman diagrams that superficially are quadratically divergent. Due to gauge invariance, the quadratic divergences of 1(a) and 1(b) cancel, so that the only remaining quadratic divergence is the tadpole graph with a single insertion of $V_{H}$ in 1(c). This diagram is local, i.e., independent of the incoming momentum, and proportional to $\int d^{2} x V_{H}$. Therefore it can be fully removed from the VPE by an appropriate no-tadpole renormalization condition.

Again by gauge invariance, the logarithmic divergences in Figs. 2(a) and 2(d) cancel, as do those of Figs. 3(a) and 3(c). Thus all we need to subtract in Eq. (7) are the divergences associated with the diagrams of Figs. 1(a)-1(c) and 3(d). The treatment of 1(c) and 3(d) is straightforward using spectral methods. We merely need to subtract the Jost functions obtained from iterating $V_{H}$ in the differential equations

$$
\begin{aligned}
& \frac{1}{\rho} \partial_{\rho} \rho \partial_{\rho} \bar{\eta}_{\ell}^{(1)}=2 t Z_{\ell}(t \rho) \partial_{\rho} \bar{\eta}_{\ell}^{(1)}+V_{H} \quad \text { and } \\
& \frac{1}{\rho} \partial_{\rho} \rho \partial_{\rho} \bar{\eta}_{\ell}^{(2)}=2 t Z_{\ell}(t \rho) \partial_{\rho} \bar{\eta}_{\ell}^{(2)}+V_{H} \bar{\eta}_{\ell}^{(1)} .
\end{aligned}
$$

Integrating these differential equations with the boundary conditions $\lim _{\rho \rightarrow \infty} \bar{\eta}_{\ell}^{(1,2)}(\rho)=0$ and $\lim _{\rho \rightarrow \infty} \frac{d}{d \rho} \bar{\eta}_{\ell}^{(1,2)}(\rho)=0$, we obtain the subtracted result

$$
\left[\nu_{\ell}(t)\right]_{1 c), 3 d)}=\lim _{\rho \rightarrow 0}\left\{\ln \left(\bar{\eta}_{\ell}\right)-\bar{\eta}_{\ell}^{(1)}-\bar{\eta}_{\ell}^{(2)}+\frac{1}{2}\left(\bar{\eta}_{\ell}^{(1)}\right)^{2}\right\} .
$$

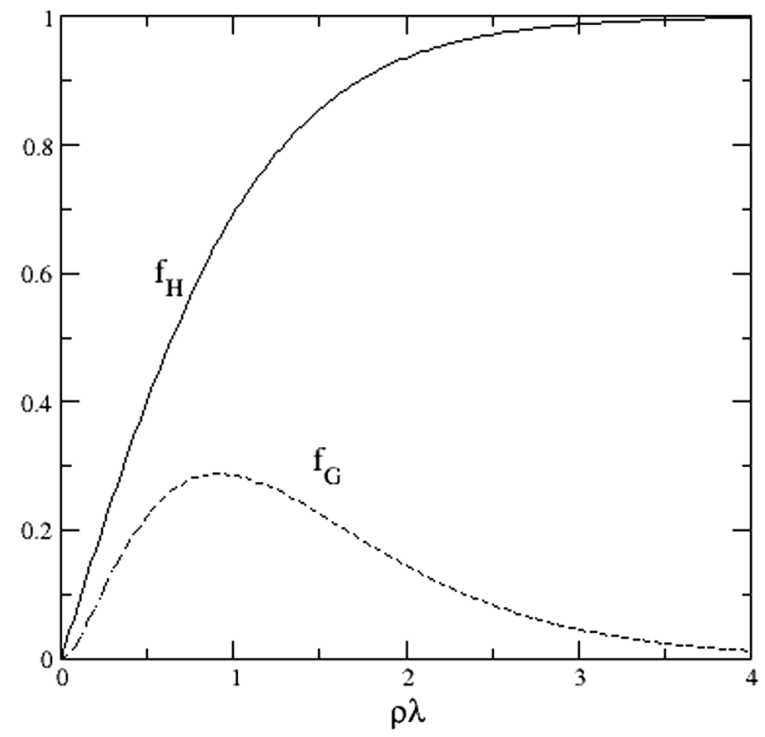

Next we explain how to complete a full set of subtractions corresponding to the remaining divergences. However, in the next section we will show a shortcut that will bypass these subtractions in favor of a simpler limiting function, which we will then use in the context of the singular vortex background in Secs. VI and VII. Diagram 1(a) has a single insertion of $\left(f_{G} / \rho\right)^{2}$ and the corresponding order of the Jost solution is given by the differential equation

$$
\frac{1}{\rho} \partial_{\rho} \rho \partial_{\rho} \bar{\eta}_{\ell}^{(3)}=2 t Z_{\ell}(t \rho) \partial_{\rho} \bar{\eta}_{\ell}^{(3)}+\left(\frac{f_{G}}{\rho}\right)^{2} .
$$

To obtain the Jost solution representing diagram 1(b), which has two distinct insertions of $2 \ell f_{G} / \rho^{2}$, we need to solve a set of coupled differential equations

$\frac{1}{\rho} \partial_{\rho} \rho \partial_{\rho} \bar{\eta}_{\ell}^{(4)}=2 t Z_{\ell}(t \rho) \partial_{\rho} \bar{\eta}_{\ell}^{(4)}-\frac{2 \ell}{\rho^{2}} f_{G} \quad$ and

$\frac{1}{\rho} \partial_{\rho} \rho \partial_{\rho} \bar{\eta}_{\ell}^{(5)}=2 t Z_{\ell}(t \rho) \partial_{\rho} \bar{\eta}_{\ell}^{(5)}-\frac{2 \ell}{\rho^{2}} f_{G} \bar{\eta}_{\ell}^{(4)}$.

Again, these differential equations are solved subject to the boundary conditions $\lim _{\rho \rightarrow \infty} \bar{\eta}_{\ell}^{(3,4,5)}(\rho)=0$ and $\lim _{\rho \rightarrow \infty} \frac{d}{d \rho} \bar{\eta}_{\ell}^{(3,4,5)}(\rho)=0$. Putting things together, the integral in Eq. (7) is rendered finite by using

$$
\begin{aligned}
{\left[\nu_{\ell}(t)\right]_{n}=} & \lim _{\rho \rightarrow 0}\left\{\ln \left(\bar{\eta}_{\ell}\right)-\bar{\eta}_{\ell}^{(1)}-\bar{\eta}_{\ell}^{(2)}+\frac{1}{2}\left(\bar{\eta}_{\ell}^{(1)}\right)^{2}-\bar{\eta}_{\ell}^{(3)}\right. \\
& \left.-\bar{\eta}_{\ell}^{(4)}-\bar{\eta}_{\ell}^{(5)}+\frac{1}{2}\left(\bar{\eta}_{\ell}^{(4)}\right)^{2}\right\} .
\end{aligned}
$$

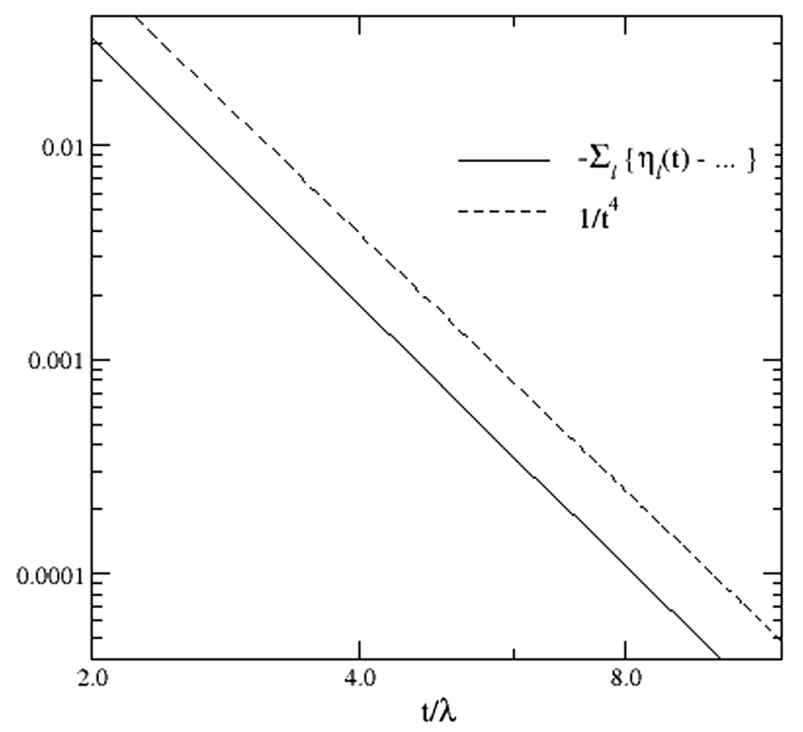

FIG. 4. Left: regular vortex background profile functions; right: double logarithmic plot of $\sum_{\ell}\left[\eta_{\ell}(t)\right]_{n}$ from this background compared to a power behavior, both with the horizontal axis expressed in units of $\lambda=m / \sqrt{2}$. 
Note that we have included $\bar{\eta}_{\ell}^{(4)}$ in the subtraction even though $\sum_{\ell=-L}^{L} \bar{\eta}_{\ell}^{(4)}=0$ because it may be advantageous numerically by avoiding summing large numbers.

Before discussing results, a remark on the numerical simulation is in order. Scattering calculations with radial symmetry separate the regular and irregular solutions at the origin. In the $\ell=0$ channel, the irregular solution diverges like a logarithm, while the regular one is constant. Numerically these two behaviors are difficult to disentangle. We therefore integrate the differential equations for $\ell=0$ down to various values in the regime $\rho_{\min }=10^{-20} \ldots 10^{-50} / m$ and fit $c_{0}+c_{1} / \ln \left[\rho_{\min } / m\right]+$ $c_{2} / \ln ^{2}\left[\rho_{\min } / m\right]$. We then make sure that the final result, $c_{0}$, is stable under further variation of $\rho_{\min }$.

In Fig. 4 we display both a sample regular vortex background and the resulting integrand $\sum_{\ell}\left[\eta_{\ell}(t)\right]_{n}$ for the VPE. Obviously, that integrand quickly approaches zero like $1 / t^{4}$, which suffices to make the integral in Eq. (7) converge. Hence we have correctly implemented the divergence structure deduced from the Feynman diagrams in the scattering calculation.

Surprisingly

$$
\begin{aligned}
& \lim _{L \rightarrow \infty} \sum_{\ell=-L}^{L} \lim _{\rho \rightarrow 0}\left\{\bar{\eta}_{\ell}^{(3)}+\bar{\eta}_{\ell}^{(4)}+\bar{\eta}_{\ell}^{(5)}-\frac{1}{2}\left(\bar{\eta}_{\ell}^{(4)}\right)^{2}\right\} \\
& \rightarrow \int_{0}^{\infty} \frac{d \rho}{\rho} f_{G}^{2}(\rho) \quad \text { as } t \rightarrow \infty
\end{aligned}
$$

signaling a quadratic divergence. The integral is the coefficient of the quadratic divergence arising when the regularization procedure is not gauge invariant, which here is a consequence of the fact that the Jost function is not gauge invariant. We will describe how to fix this problem in the discussion below.

\section{FOURIER TRANSFORMS AND FEYNMAN DIAGRAMS}

As far as the gauge field part of the background is concerned, we have argued above that even though there are many divergent diagrams, only the divergences from 1(a) and 1(b) will remain once all divergent diagrams are combined with a gauge invariant regularization.

Let us first comment on the origin of Eq. (16). In a covariant, but not necessarily gauge invariant regularization, diagrams 1(a) and 1(b) contribute

$$
\begin{aligned}
& \int d^{4} x A_{\mu}(x) A_{\nu}(x) \int \frac{d^{4} l}{(2 \pi)^{4}} \\
& \times\left[\frac{g^{\mu \nu}}{l^{2}-m^{2}+\mathrm{i} 0^{+}}-\frac{1}{2} \frac{4 l^{\mu} l^{\nu}}{\left(l^{2}-m^{2}+\mathrm{i} 0^{+}\right)^{2}}\right]
\end{aligned}
$$

as the leading divergence in the effective action. In dimensional regularization the two terms cancel. However, using a sharp cutoff $\Lambda$ in Euclidean space yields

$$
\begin{aligned}
& \frac{\Omega_{D}}{D} \frac{\Lambda^{D}}{\Lambda^{2}+m^{2}} \\
& \quad-\Omega_{D} \int_{0}^{\Lambda} d l\left[\frac{l^{D}}{D} \frac{d}{d l}\left(\frac{1}{l^{2}+m^{2}}\right)+\frac{2}{D} \frac{l^{D+1}}{\left(l^{2}+m^{2}\right)^{2}}\right]
\end{aligned}
$$

times $g^{\mu \nu}$ for the loop momentum integral, where $\Omega_{D}$ is the $D$-dimensional solid angle. The integrand indeed vanishes for any $D$, but the surface term does not vanish for $D \geq 2$ as the regulator is removed. ${ }^{2}$ With $A_{\mu}(x) A^{\mu}(x)=-\left(\frac{f_{G}}{\rho}\right)^{2}$ we observe that the quadratic divergence for $D=2$ is proportional to the coefficient given in Eq. (16).

In dimensional regularization (with $D$ space time dimensions) the diagrams 1(a) and 1(b) contribute

$$
\begin{aligned}
E_{\mathrm{VPE}}^{(A)}= & \Gamma\left(1-\frac{D}{2}\right)\left(\frac{4 \pi \mu^{2}}{m^{2}}\right)^{2-D / 2}\left(\frac{m}{4 \pi}\right)^{2} \int \frac{d^{2} p}{(2 \pi)^{2}} \tilde{\boldsymbol{A}}(\boldsymbol{p}) \\
& \cdot \tilde{\boldsymbol{A}}(-\boldsymbol{p}) \int_{0}^{1} d x\left\{1-\left[1+x(1-x) \frac{\boldsymbol{p}^{2}}{m^{2}}\right]^{D / 2-1}\right\}
\end{aligned}
$$

to the VPE of the vortex per unit length. The derivation of Eq. (19) used that $\partial_{\mu} A^{\mu}=0$ translates into $\boldsymbol{p} \cdot \tilde{\boldsymbol{A}}(\boldsymbol{p})=0$. The renormalization scale $\mu$ will eventually be eliminated by appropriate renormalization conditions. The pole at $D=2$, corresponding to the superficial quadratic divergence, has zero residue and we may thus analytically continue to $D=4-2 \epsilon$ without adding a counterterm for this divergence. The logarithmic divergence in Eq. (19) becomes

$$
\begin{aligned}
\left.E_{\mathrm{VPE}}^{(A)}\right|_{\text {div. }} & =\frac{1}{6 \epsilon(4 \pi)^{2}} \int \frac{d^{2} p}{(2 \pi)^{2}} \boldsymbol{p}^{2} \tilde{\boldsymbol{A}}(\boldsymbol{p}) \cdot \tilde{\boldsymbol{A}}(-\boldsymbol{p}) \\
& =\frac{1}{12 \epsilon(4 \pi)^{2}} \int d^{2} x F_{\mu \nu} F^{\mu \nu} .
\end{aligned}
$$

Using the inverse Fourier transformation to write a coordinate space integral is actually essential. As we will see later, momentum space integrals above may not be well defined for a singular vector potential, while the field strength tensor only contains the magnetic field, which is nonsingular. In a full theory the ultraviolet divergence in Eq. (20) is compensated by wave-function renormalization of the dynamical gauge field. We therefore refer to this divergence as wave-function renormalization.

\footnotetext{
${ }^{2}$ Dimensional regularization takes $D$ small enough such that the surface term tends to zero as $\Lambda \rightarrow \infty$.
} 
In the next step we translate the logarithmic divergence of Eq. (20) into the large $t$-behavior of $\nu(t)$. We write, in terms of an arbitrary mass scale $M$,

$$
\begin{aligned}
\frac{1}{\epsilon(4 \pi)^{2}} & =-\left.\mathrm{i} \int \frac{d^{4} l}{(2 \pi)^{4}} \frac{1}{\left(l^{2}-M^{2}+\mathrm{i} 0^{+}\right)^{2}}\right|_{\text {div. }} \\
& =\left.\frac{1}{8 \pi^{2}} \int \frac{l^{2} d l}{\sqrt{l^{2}+M^{2}}}\right|_{\text {div. }},
\end{aligned}
$$

so that we may formally identify

$\left.E_{\mathrm{VPE}}^{(A)}\right|_{\text {div. }}=\left.\frac{1}{96 \pi^{2}}\left[\int d^{2} x F_{\mu \nu} F^{\mu \nu}\right] \int \frac{l^{2} d l}{\sqrt{l^{2}+M^{2}}}\right|_{\text {div. }}$.

Hence with only gauge field insertions (i.e., $V_{H}=0$ ), the unsubtracted integrand in Eq. (7) has the asymptotic behavior

$[\nu(t)]_{0}-\int_{0}^{\infty} \frac{d \rho}{\rho} f_{G}^{2}(\rho) \rightarrow \nu_{\text {l.f. }}(t)=\frac{1}{t^{2}} \frac{1}{12} \int_{0}^{\infty} \rho d \rho\left(\frac{f_{G}^{\prime}(\rho)}{\rho}\right)^{2}$

as $t \rightarrow \infty$, where $F_{\mu \nu} F^{\mu \nu}=2\left(\frac{f_{G}^{\prime}(\rho)}{\rho}\right)^{2}$ for the vortex. With $V_{H} \neq 0$, the limiting function (l.f.) should also be approached when the divergences arising from $V_{H}$ are removed, i.e., by using Eq. (12) instead of Eq. (15) and subtracting the constant of Eq. (16).

We can restate the above analysis of the ultraviolet behavior by supplementing Eq. (16) with the next-toleading order term

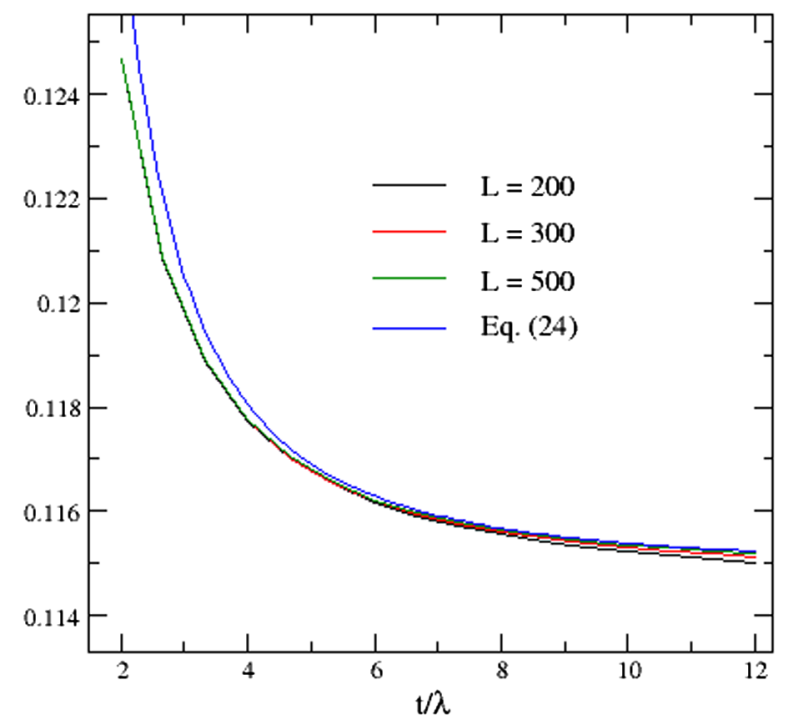

$$
\begin{gathered}
\lim _{L \rightarrow \infty} \sum_{\ell=-L}^{L} \lim _{\rho \rightarrow 0}\left\{\bar{\eta}_{\ell}^{(3)}+\bar{\eta}_{\ell}^{(4)}+\bar{\eta}_{\ell}^{(5)}-\frac{1}{2}\left(\bar{\eta}_{\ell}^{(4)}\right)^{2}\right\} \\
\rightarrow \int_{0}^{\infty} \frac{d \rho}{\rho} f_{G}^{2}(\rho)+\frac{1}{12 t^{2}} \int_{0}^{\infty} \rho d \rho\left(\frac{f_{G}^{\prime}(\rho)}{\rho}\right)^{2}
\end{gathered}
$$

as $t \rightarrow \infty$. We numerically calculate this asymptotic expression in Fig. 5. For this calculation we need to terminate the sum on the left-hand side at some large but finite value. From the left panel of this figure we see that the leading terms of the Born series nicely approach the expected asymptotic form for moderate momenta $t$. However, closer inspection (right panel) shows that even for values as large as $L=500$, the asymptotic form is not exactly reached. Rather, the difference between the sum and the asymptotic form increases with $t$. For this reason we use various (large) values for $L$ and fit an inverse polynomial

$$
\begin{aligned}
& \sum_{\ell=-L}^{L} \lim _{\rho \rightarrow 0}\left\{\bar{\eta}_{\ell}^{(3)}+\bar{\eta}_{\ell}^{(4)}+\bar{\eta}_{\ell}^{(5)}-\frac{1}{2}\left(\bar{\eta}_{\ell}^{(4)}\right)^{2}\right\} \\
& =c_{0}+\frac{c_{1}}{L}+\frac{c_{2}}{L^{2}}+\ldots
\end{aligned}
$$

so that $c_{0}$ is the $L \rightarrow \infty$ extrapolation. Indeed, we see from the right panel of Fig. 5 that this extrapolation perfectly matches the asymptotic form extracted from the analysis of the Feynman diagrams. This result shows that we do not miss any subleading logarithmic divergences when we subtract the constant in Eq. (16). For regular profiles, the numerical effect of this extrapolation is actually very marginal. However, we will see later that for singular vortex profiles this extrapolation is essential and its omission leads to incorrect results.

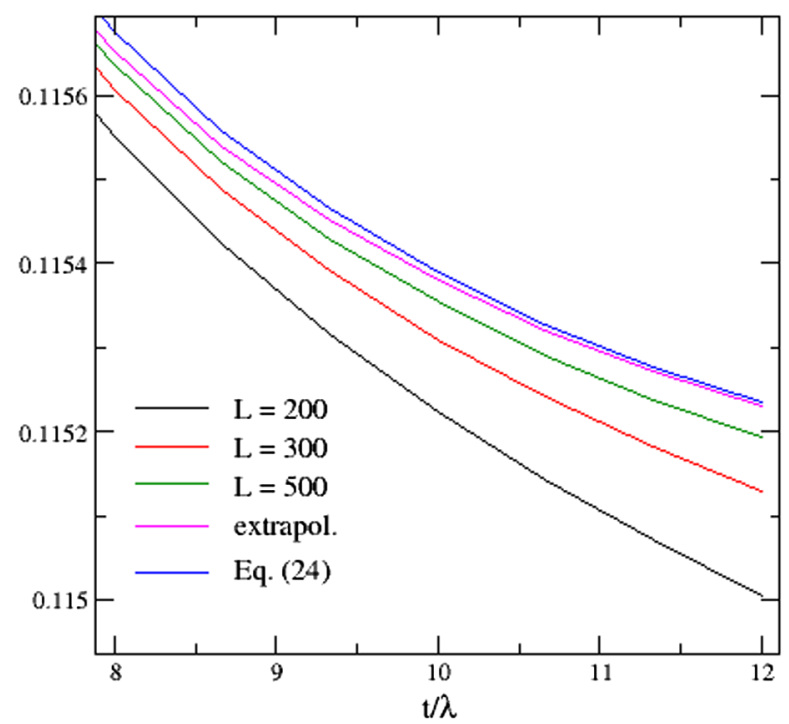

FIG. 5. Comparison of Born approximation and asymptotics extracted from Feynman diagrams, both with the horizontal axis expressed in units of $\lambda=m / \sqrt{2}$. 
In further preparation for the consideration of singular gauge profiles, it is illuminating to consider the finite part in Eq. (19). In $\overline{\mathrm{MS}}$ renormalization, it reads

$$
\begin{aligned}
\bar{E}_{\mathrm{VPE}}^{(A)} & =-\left(\frac{m}{4 \pi}\right)^{2} \int \frac{d^{2} p}{(2 \pi)^{2}} \tilde{\boldsymbol{A}}(\boldsymbol{p}) \cdot \tilde{\boldsymbol{A}}(-\boldsymbol{p}) \int_{0}^{1} d x\left[1+x(1-x) \frac{\boldsymbol{p}^{2}}{m^{2}}\right] \ln \left[1+x(1-x) \frac{\boldsymbol{p}^{2}}{m^{2}}\right] \\
& =-\frac{m^{2}}{8 \pi} \int_{0}^{\infty} p d p a^{2}(p) \int_{0}^{1} d x\left[1+x(1-x) \frac{p^{2}}{m^{2}}\right] \ln \left[1+x(1-x) \frac{p^{2}}{m^{2}}\right],
\end{aligned}
$$

where $a(p)=\int_{0}^{\infty} d \rho f_{G}(\rho) J_{1}(\rho p)$ (which indeed exists even for a singular background). Straightforward substitution of Eq. (3) fails for profiles with $f_{G}(0) \neq 0$, as can be seen already from the leading $\boldsymbol{p}^{2}$ term. Integration by parts of $\frac{d}{d \rho} J_{0}(p \rho)=$ $-p J_{1}(p \rho)$ and the completeness relation for the Bessel functions yield

$$
\int_{0}^{\infty} p d p p^{2} a^{2}(p)=f_{G}^{2}(0) \int_{0}^{\infty} p d p+2 f_{G}(0) \int_{0}^{\infty} p d p\left[\int_{0}^{\infty} d \rho J_{0}(p \rho) f_{G}^{\prime}(\rho)\right]+\int_{0}^{\infty} \rho d \rho\left(\frac{f_{G}^{\prime}(\rho)}{\rho}\right)^{2} .
$$

The first two integrals diverge, while the last term is the correct local integral $\int \frac{d^{2} x}{4 \pi} F_{\mu \nu} F^{\mu \nu}$. In order to compute Eq. (26) for a singular gauge profile, we first note that the Feynman parameter integral in Eq. (26) vanishes for $\boldsymbol{p}^{2}=0$, so that

$$
\bar{E}_{\mathrm{VPE}}^{(A)}=-\left(\frac{1}{4 \pi}\right)^{2} \int \frac{d^{2} p}{(2 \pi)^{2}} \boldsymbol{p}^{2} \tilde{\boldsymbol{A}}(\boldsymbol{p}) \cdot \tilde{\boldsymbol{A}}(-\boldsymbol{p}) \int_{0}^{1} d x\left[\frac{m^{2}}{\boldsymbol{p}^{2}}+x(1-x)\right] \ln \left[1+x(1-x) \frac{\boldsymbol{p}^{2}}{m^{2}}\right]
$$

is well defined. Next we observe that (formally)

$$
\int \frac{d^{4} p}{(2 \pi)^{4}} p_{\mu} \tilde{A}_{\nu}(p) p^{\mu} \tilde{A}^{\nu}(-p) g(p)=\frac{1}{2} \int \frac{d^{4} p}{(2 \pi)^{4}} \tilde{F}_{\mu \nu}(p) \tilde{F}^{\mu \nu}(-p) g(p)
$$

again using $\partial_{\mu} A^{\mu}=0$. We then define

$$
b(p)=-\int_{0}^{\infty} d \rho f_{G}^{\prime}(\rho) J_{0}(p \rho)
$$

to find

$$
\bar{E}_{\mathrm{VPE}}^{(A)}=-\frac{1}{8 \pi} \int_{0}^{\infty} p d p b^{2}(p) \int_{0}^{1} d x\left[\frac{m^{2}}{p^{2}}+x(1-x)\right] \ln \left[1+x(1-x) \frac{p^{2}}{m^{2}}\right]
$$

For regular profiles we have numerically verified that Eqs. (26) and (31) yield identical results. However, Eq. (31) is also well defined for singular vortex profiles.

\section{SINGULAR BACKGROUND}

The standard procedure outlined in Sec. IV fails when $f_{G}(0) \neq 0$. The situation is even worse than Eq. (27) suggests because neither the Born approximation nor the Fourier transform of the radial function $\left[f_{G}^{2}-2 \ell f_{G}\right] / \rho^{2}$ exist. Yet we expect

$$
\begin{aligned}
{[\nu(t)]_{H}=} & \left.\lim _{L \rightarrow \infty} \sum_{\ell=-L}^{L}\left\{\ln \left(\bar{\eta}_{\ell}\right)-\bar{\eta}_{\ell}^{(1)}-\bar{\eta}_{\ell}^{(2)}+\frac{1}{2}\left(\bar{\eta}_{\ell}^{(1)}\right)^{2}\right\}\right|_{\rho=\rho_{\min }} \\
& -\int_{\rho_{\min }}^{\infty} \frac{d \rho}{\rho} f_{G}^{2}(\rho)
\end{aligned}
$$

to approach $\nu_{\text {l.t. }}(t)$ as $\rho_{\min } \rightarrow 0$ and $t \rightarrow \infty$, to properly produce the logarithmic divergence. Essentially we have subtracted the ultraviolet divergences associated with the scalar potential as in Eq. (12) and subtracted the constant, which formally does not contribute to the VPE, needed to maintain the analytical properties of the (summed logarithm of the) Jost function. The above analysis refers to the scattering data for imaginary momenta, but we have also computed the Jost function for real momenta. In that case the subtracted constant emerges as the modulus of the Jost function and thus does not contribute to the VPE which, on the real axis, involves only the phase of the Jost function and the bound state energies.

We have numerically verified that the regulator $\rho_{\min }$ in that integral must be identical to the end point of the differential equation. Surprisingly, we also find numerically 
that, with this regularization installed, the sensitivity on $\rho_{\text {min }}$ is mitigated.

To construct the limiting function that will allow us to implement the final logarithmic renormalization, we require a background potential that has the same logarithmic divergence in its second order Born term. For this purpose we introduce $V_{f}=\frac{3 m^{2}}{2}\left(\tanh ^{2}(\rho)-1\right)$, which couples to the scalar quantum fluctuations via Feynman diagrams like 1(c) and 3(d). All we require are the solutions to Eq. (11) with $V_{H}$ replaced by $V_{f}$. Calling those solutions $\bar{\zeta}_{\ell}^{(1,2)}(\rho)$, we find the second order contribution

$\bar{\nu}^{(2)}(t)=\lim _{L \rightarrow \infty} \sum_{\ell=0}^{L}\left(2-\delta_{\ell 0}\right) \lim _{\rho \rightarrow 0}\left[\bar{\zeta}_{\ell}^{(2)}(\rho)-\frac{1}{2}\left(\bar{\zeta}_{\ell}^{(1)}(\rho)\right)^{2}\right]$.

Similarly to Eq. (23), it produces the limiting function

$\bar{\nu}^{(2)}(t) \rightarrow \bar{\nu}_{\text {l.f. }}(t)=-\frac{1}{4 t^{2}} \int_{0}^{\infty} \rho d \rho V_{f}^{2} \quad$ as $t \rightarrow \infty$.

Using this quantity we compute the finite scattering data component of the VPE as

$$
\begin{aligned}
E_{\mathrm{VPE}}^{\text {scat. }} & =\frac{1}{2 \pi} \int_{m}^{\infty} t d t\left[[\nu(t)]_{H}-c_{B} \bar{\nu}^{(2)}(t)\right] \\
\text { with } \quad c_{B} & =-\frac{1}{3} \frac{\int_{0}^{\infty} \rho d \rho\left(\frac{f_{G}^{\prime}(\rho)}{\rho}\right)^{2}}{\int_{0}^{\infty} \rho d \rho V_{f}^{2}} .
\end{aligned}
$$

Instead of the wave-function renormalization, Eq. (31), we have to add back the Feynman diagrams for two scalar field insertions, both the regular, $V_{H}$, and the auxiliary, $V_{f}$. In $\overline{\mathrm{MS}}$ renormalization they are combined to

$$
\begin{aligned}
\bar{E}_{\mathrm{VPE}}^{(S)}= & \frac{1}{16 \pi} \int_{0}^{\infty} p d p\left[v_{H}^{2}(p)+c_{B} v_{f}^{2}(p)\right] \\
& \times \int_{0}^{1} d x \ln \left[1+x(1-x) \frac{p^{2}}{m^{2}}\right]
\end{aligned}
$$

where $v_{H, f}(p)=\int_{0}^{\infty} \rho d \rho V_{H, f}(\rho) J_{0}(p \rho)$.

In total, the VPE for the singular background is given by

$$
E_{\mathrm{VPE}}=E_{\mathrm{VPE}}^{\mathrm{scat}}+\bar{E}_{\mathrm{VPE}}^{(S)} \text {. }
$$

\section{NUMERICAL EXPERIMENTS}

For the numerical simulations we consider a family of (singular) background profiles

$$
f_{H}(\rho)=\tanh (\alpha \rho \lambda) \quad \text { and } \quad f_{G}(\rho)=\mathrm{e}^{-(\alpha \rho \lambda)^{2}}
$$

that have shapes similar to the ANO profiles. Here $\alpha$ is a dimensionless variational parameter and $\lambda=m / \sqrt{2}$ provides a convenient energy (or, equivalently, inverse length) scale. Though the gauge potential is singular at $\rho \rightarrow 0$, the profile of the magnetic field, $\frac{f_{G}^{\prime}(\rho)}{\rho}$, has a smooth limit. To study the relative roles of the scalar and gauge potentials, we introduce the additional parameter $\beta$ and define $V_{H}=\left(3 m^{2} / 2\right) \beta\left[f_{H}^{2}(\rho)-1\right]$. Note that the model Lagrangian, Eq. (1), implies $\beta=1$. However, our main interest is to compare the scattering results based on Eq. (5) with the canonical expansion of the effective action, Eq. (9). Then, e.g., a small value of $\beta$ is an ideal means to investigate the role of the singularity in $\boldsymbol{A}$.

In order to obtain numerically stable results when integrating differential equations (8), (11), etc. we needed to develop a long-double precision FORTRAN code for an adaptive step size control in combination with a fourth order Runge-Kutta algorithm [18]. The algorithm for computing the modified Bessel function $K_{|\ell|}(t \rho)$ is an iterative process in $\ell$, which is computationally costly for large $\ell$. Some of the simulations that did not require extremely large wave numbers and angular momenta were cross-checked with computations in Mathematica. The necessity of this elaborate simulation raises doubts about the numerical results from the simpler treatment reported in Refs. $[5,6]$.

To begin with, we have to verify that Eq. (32) indeed approaches the limiting function, Eq. (23), when the imaginary momentum $t$ becomes large. For this purpose it is useful to introduce a variant of Eq. (32) without the $L \rightarrow \infty$ limit

$$
\begin{aligned}
\nu_{L}(t)= & \left.\sum_{\ell=-L}^{L}\left\{\ln \left(\bar{\eta}_{\ell}\right)-\bar{\eta}_{\ell}^{(1)}-\bar{\eta}_{\ell}^{(2)}+\frac{1}{2}\left(\bar{\eta}_{\ell}^{(1)}\right)^{2}\right\}\right|_{\rho=\rho_{\min }} \\
& -\int_{\rho_{\min }}^{\infty} \frac{d \rho}{\rho} f_{G}^{2}(\rho) .
\end{aligned}
$$

We have confirmed that the results are insensitive but not fully independent of $\rho_{\min }$ once it is taken small enough. That is, the logarithmic divergences in Eq. (39) cancel. However, there is an additional mild but not negligible sensitivity on $\rho_{\min }$ also known for regular scattering potentials [19]. It arises from separating regular and irregular solutions of the wave equation which for $\ell=0$ requires numerically distinguishing a slowly varying logarithm from a constant, while otherwise the behavior differs as $\rho_{\min }^{2|\ell|}$. As in Sec. IV we therefore extrapolate to $\rho_{\min } \rightarrow 0$ : For $|\ell| \leq 1$ and the integral in Eq. (39) we take various values for $\rho_{\min } \in$ $\left[10^{-50}, 10^{-20}\right]$ and fit $p_{0}+p_{1} / \ln \left(\rho_{\min }\right)+p_{2} / \ln ^{2}\left(\rho_{\min }\right)$ so that $p_{0}$ is our result, which indeed is stable against further changes. ${ }^{3}$

The dependence on $L$ is much more intricate. A typical result is shown in Fig. 6. At first sight (left panel) we

\footnotetext{
${ }^{3}$ No problems related with $\rho_{\min } \rightarrow 0$ have been reported in Ref. [6] which adopted the numerical procedure for integrating the radial differential equations from Ref. [5]. It appears that the discretization grid was not varied in either reference and that $\rho_{\min }$ was fixed at a value as big as about 0.001 .
} 

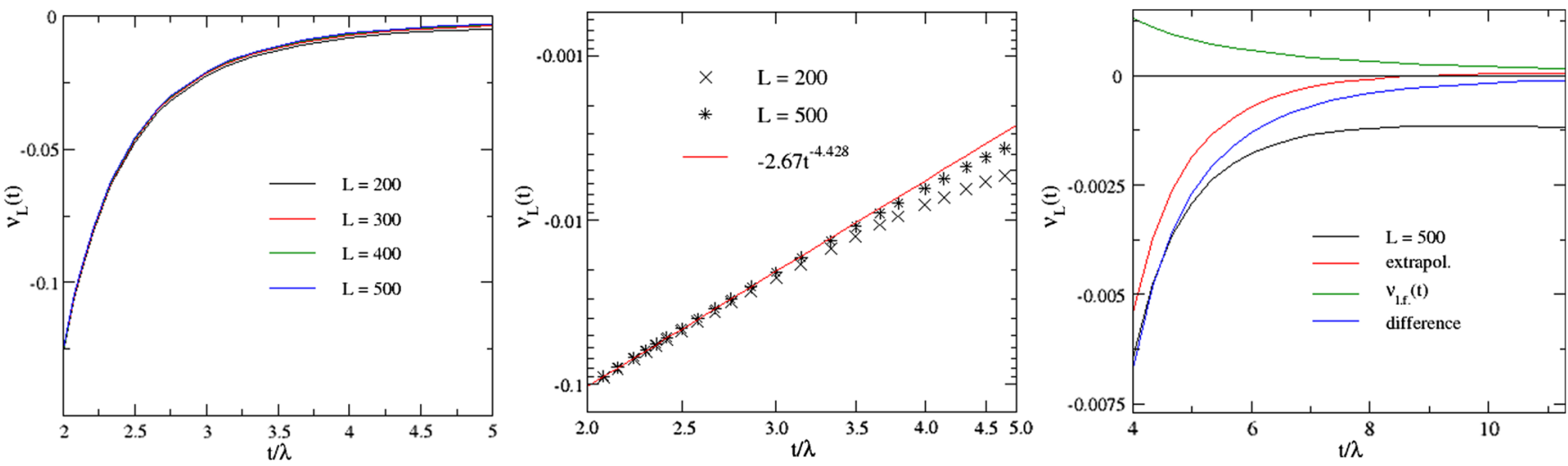

FIG. 6. Asymptotic behavior of $\nu_{L}(t)$ defined in Eq. (39) using $\alpha=0.5$ and $\beta=1.0$ in Eq. (38). Left: different maximal angular momenta $L$; center: fit showing that $\nu_{L}(t)$ falls faster than $1 / t^{2}$ for moderate values of $t$; right: extrapolation $L \rightarrow \infty$ and limiting function from Eq. (23), all with the horizontal axis expressed in units of $\lambda=m / \sqrt{2}$. Also shown is the difference between the extrapolated and limiting functions.
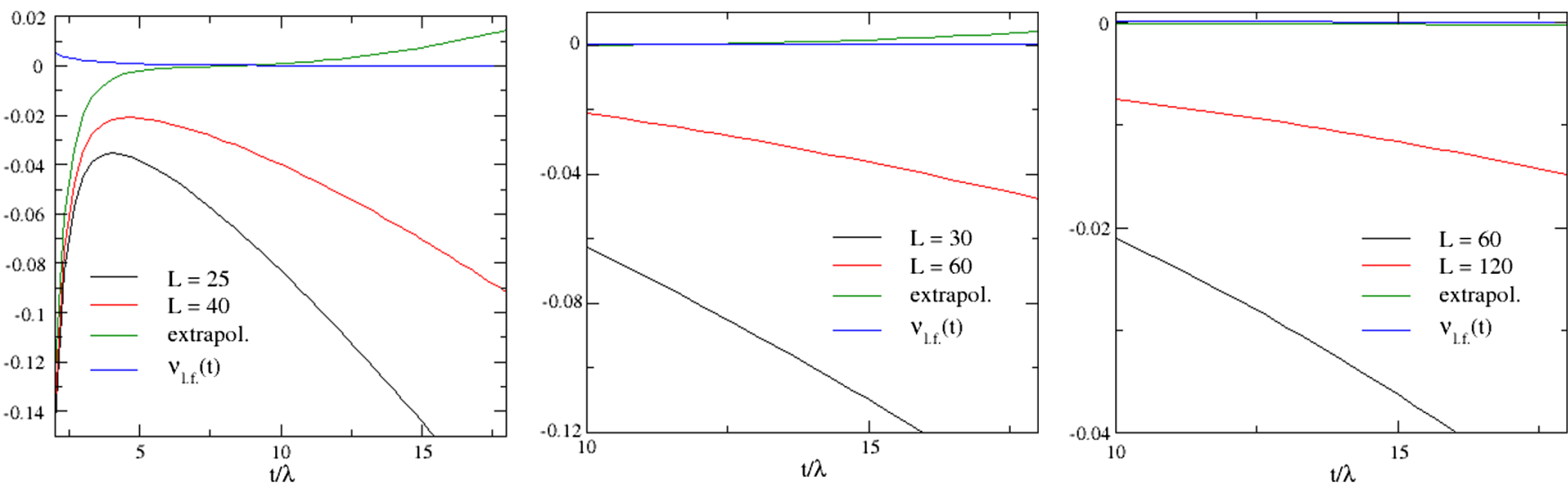

FIG. 7. Asymptotic structure of the angular momentum sum in Eq. (39) for $\alpha=0.5$ and $\beta=1.0$. Four equi-distant $L$ values between (and including) the explicitly given ones are used to match the right-hand-side as in Eq. (25). Note the different intervals for the horizontal axes, which are given in units of $\lambda=m / \sqrt{2}$.

observe a reasonably convergent function as $L$ becomes very big. The graphs indicate that the sums indeed approach zero at large $t$. Yet there are problems with those curves. The double-logarithmic plot in the center panel of Fig. 7 strongly suggests that $\nu_{L}(t)$ approaches zero much faster than $1 / t^{2}$ for moderate $t$. Hence using $\nu_{L}(t)$ instead of $[\nu(t)]_{n}$ in Eq. (7) would produce a finite result without wave-function renormalization of the gauge field. ${ }^{4}$ Alternatively, we might be missing a subleading logarithmic divergence. This, however, would invalidate our results for regular profiles. Either way, the observed behavior of $\nu_{L}(t)$ looks like a clear contradiction of renormalizability. Even worse, $\nu_{L}(t)$ seems to be negative for all values of $t$, while the limiting function, Eq. (23), is positive as we observed for regular profiles. It is therefore unavoidable to investigate the large $t$ and large $L$ behavior in more detail. The right panel of Fig. 6 also shows the result of an

\footnotetext{
${ }^{4} \mathrm{We}$ remark that a similar behavior was observed for the fermion VPE of a vortex [7].
}

extrapolation for $\nu_{L}(t)$ as in Eq. (25). We observe that the difference between the extrapolated and the $L=500$ curves increases with $t$. Eventually the extrapolated curve indeed crosses zero and approaches the limiting function such that the difference decays like $1 / t^{4}$. We observe this behavior also when fitting $\nu_{L}(t)$ for $t / \lambda \in[4,8]$. For $L=$ 500 we find $\nu_{500}(t) \approx-0.039 \lambda^{2} / t^{2}-0.966 \lambda^{4} / t^{4}(0.995)$, while the extrapolated function follows $\nu_{\infty}(t) \approx 0.023 \lambda^{2} /$ $t^{2}-1.750 \lambda^{4} / t^{4}(1.000)$; the data in parentheses are the correlation coefficients of the respective fits. For $t / \lambda \in$ $[8,14]$ we find $\nu_{\infty}(t) \approx 0.021 \lambda^{2} / t^{2}-1.640 \lambda^{4} / t^{4}(1.000)$, while $\nu_{500}(t)$ departs from zero for $t>10 \lambda$ and we cannot reasonably approximate it with a polynomial of inverse momenta in this regime. Recalling that $\nu_{\text {l.f. }}(t) \approx 0.021 \lambda^{2} / t^{2}$ we conclude that $\nu_{\text {l.f. }}(t)$ is not matched for any finite $L$ and that the extrapolation to infinitely large angular momenta is essential.

For any fixed value of $L$, there is a critical momentum $t$ above which $\nu_{L}(t)$ deviates from the convergence pattern 
suggested by the left panel of Fig. 6, where it may even acquire a negative slope as a function of $t$. In our numerical simulation we were able to generate stable results for $L \lesssim 600$. However, even that many partial waves were not large enough to obtain any positive result for $\nu_{L}(t)$ when using $\alpha=0.5$. Said another way, without the extrapolation we get nowhere near the limiting function. This extrapolation is thus essential for the singular background, because otherwise $\nu_{L}(t)$ deviates significantly from the limiting function at moderate momenta (unlike the case of regular backgrounds). This deviation will only be overcome at large imaginary momenta $t$, which in turn requires us to include extremely large angular momenta.

It is worthwhile to ask whether such large $L$ values are needed to obtain a reliable extrapolation. The results of the corresponding simulations are shown in Fig. 7. Obviously,
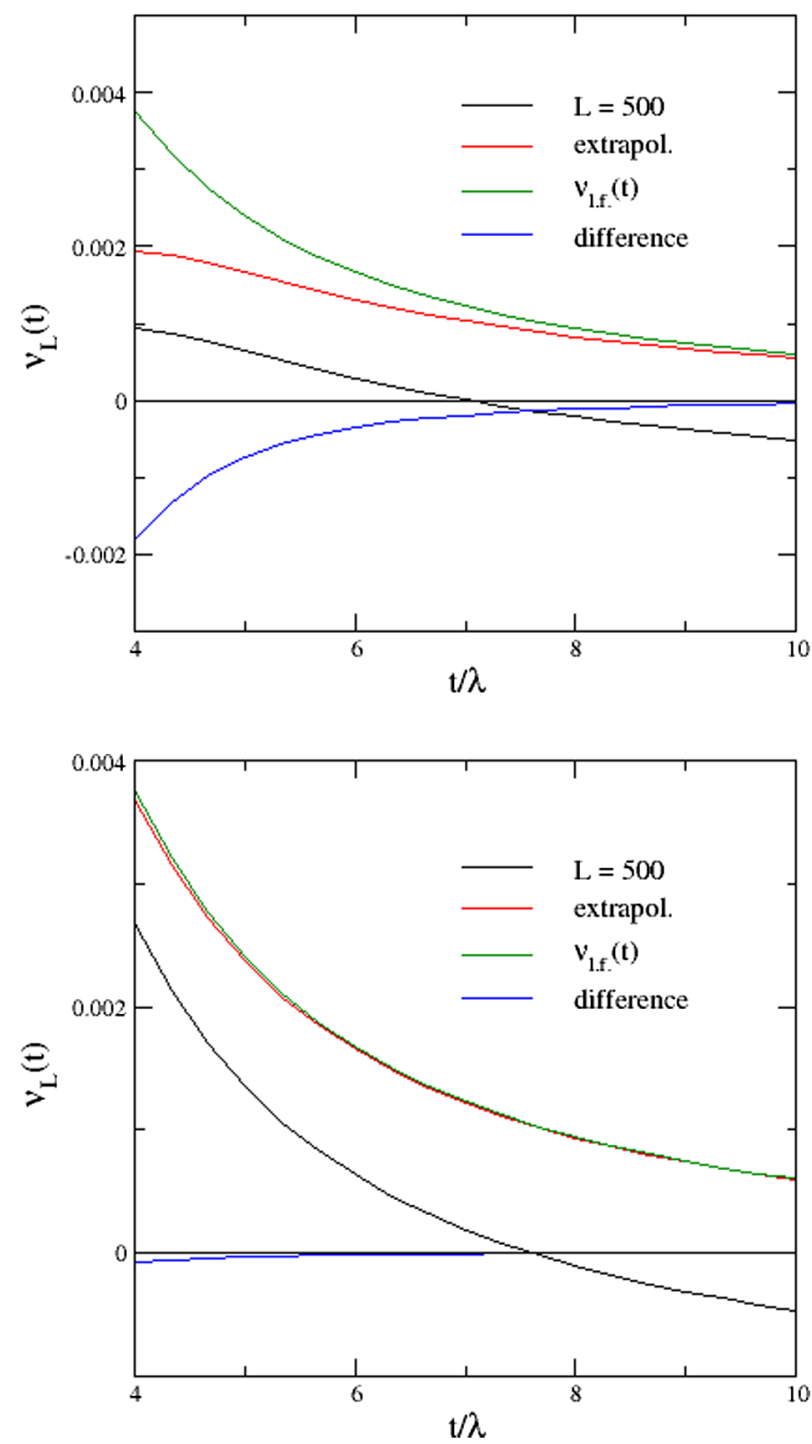

extrapolations that fit the right-hand side of Eq. (25) in the vicinity of $L=50$ do not match the limiting function and actually produce an asymptotic behavior that departs from zero already at moderate momenta. An extrapolation based on results from $L \sim 100$ appears to do better, but a closer inspection also reveals that the extrapolation in the right panel in Fig. 7 has a maximum at $t \approx 7 \lambda$ and departs from zero for larger momenta. These results cast doubt on the numerical simulations of Ref. [6], which employed $L \in[25,35]$ for the extrapolation. One may argue that the numerical error that arises from not matching the correct asymptotic behavior might be negligible and one could simply omit the contribution to the VPE arising from moderate and large $t$. Note, however, that there is an additional factor $t$ in the integrand of Eq. (7), and that the value of $t$ at which that integral may eventually be truncated
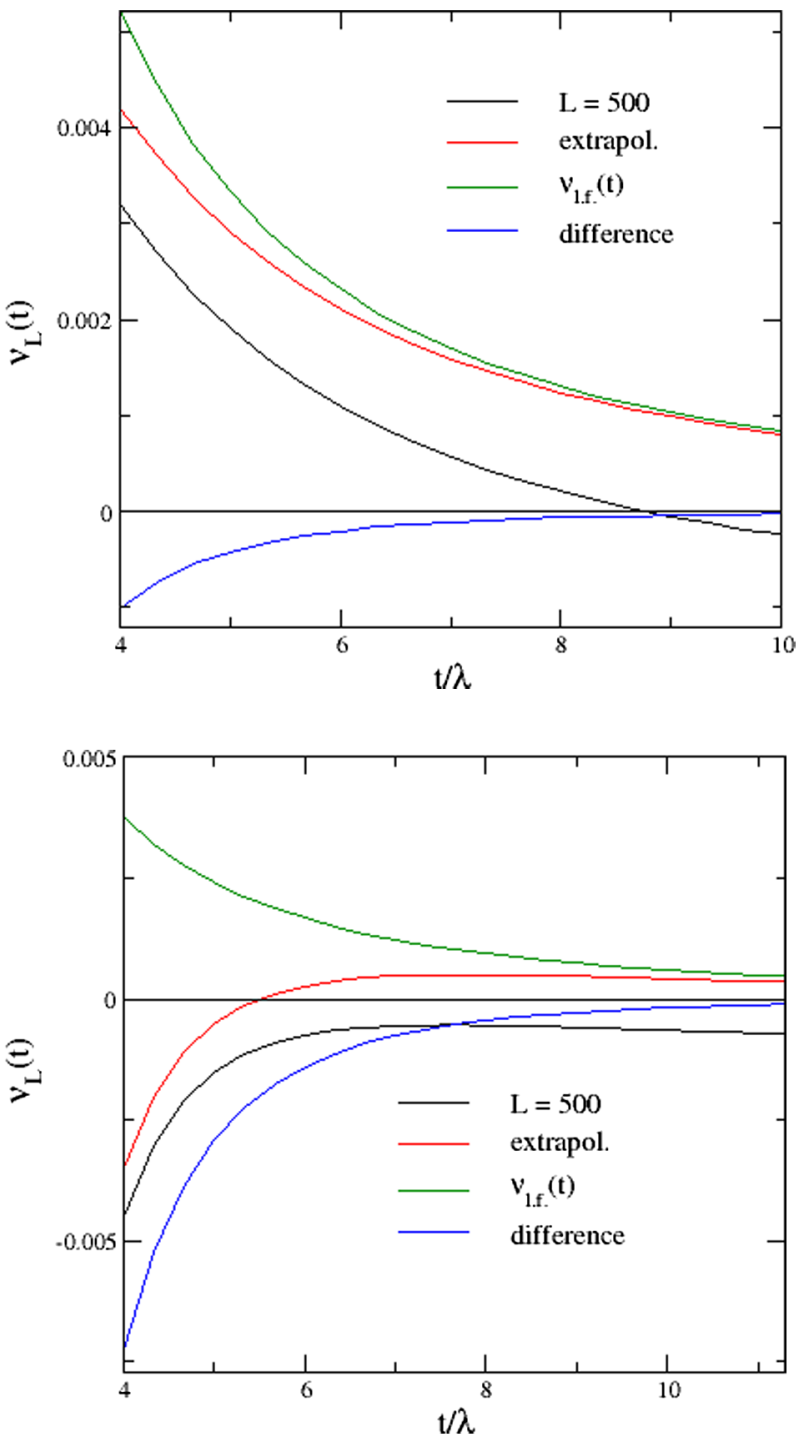

FIG. 8. Same as right panel of Fig. 6 for several variational parameters, cf. Eq. (38). Top row: $\beta=1$ with $\alpha=0.85$ (left) and $\alpha=1.0$ (right). Bottom row: $\alpha=0.85$ with $\beta=0.5$ (left) and $\beta=1.5$ (right). The horizontal axis in each case is expressed in units of $\lambda=m / \sqrt{2}$. 
TABLE I. The vacuum polarization energy (VPE) per unit length (in units of $\lambda^{2}=m^{2} / 2$ ) and its components for several variational parameters, cf. Eq. (38) in the $\overline{\mathrm{MS}}$ renormalization scheme. Also presented is the second order gauge field contribution of Eq. (31).

\begin{tabular}{lccccc}
\hline \hline$\alpha$ & $\beta$ & $E_{\mathrm{VPE}}^{\text {scat. }} / \lambda^{2}$ & $\bar{E}_{\mathrm{VPE}}^{(S)} / \lambda^{2}$ & $E_{\mathrm{VPE}} / \lambda^{2}$ & $\bar{E}_{\mathrm{VPE}}^{(A)} / \lambda^{2}$ \\
\hline 0.50 & 1.0 & -0.0862 & 0.0061 & -0.0801 & -0.0017 \\
0.85 & 1.0 & -0.0184 & 0.0053 & -0.0131 & -0.0051 \\
1.00 & 1.0 & -0.0089 & 0.0050 & -0.0039 & -0.0072 \\
0.85 & 1.5 & -0.0917 & 0.0127 & -0.0790 & -0.0051 \\
0.85 & 0.5 & -0.0002 & 0.0010 & 0.0008 & -0.0051 \\
\hline \hline
\end{tabular}

can only be determined a posteriori. The essential criterion is that there is a significantly large interval along the $t$ axis in which the numerical simulation produces a $1 / t^{4}$ behavior for the difference between the extrapolation and the limiting function.

We show the asymptotic behavior for different profiles in Fig. 8. The behavior determined by the ultraviolet analysis is well hidden when the scalar potential is very attractive, either by being wide ( small $\alpha$ ) or deep (large $\beta$ ). The weaker the scalar potential, the more closely the extrapolation of $\nu_{L}(t)$ follows the limiting function. For none of the considered cases did we observe that the large but fixed $L$ result matched the limiting function.

The strong enhancement at small $t$ is well fitted by the integrable function $A_{1}+A_{2} \ln \left(\frac{t-\mu}{\lambda}\right)$ with constants $A_{1,2}$ and $\mu \lesssim m$. We associate this behavior with the second order Born terms that are subtracted in $\nu_{L}(t)$ for the scalar potential. It is thus not surprising (and actually it is required) that we observe a similar behavior for $\bar{\nu}^{(2)}(t)$, as defined in Eq. (34). When computing the VPE, we solve the relevant differential equations starting at $t=1.45 \lambda$ and fit such a logarithmic function directly to the integrand of Eq. (35) to obtain the contribution from the interval $t / \lambda \in[\sqrt{2}, 1.45]$. That interval typically contributes about $5 \%$ to the full integral. Finally, we are in the position to give numerical results for the VPE in Table I.

Though the main purpose of this calculation has been to show the feasibility of this systematic approach, we also see that the VPE varies strongly with the strength of the scalar potential, which dominates the VPE. This result is corroborated by the small contribution of the renormalized second order Feynman diagrams of Figs. 1(a) and 1(b) listed in the last column of Table I.

\section{SUMMARY AND OUTLOOK}

We have resolved a number of technical subtleties that are encountered when applying the efficient spectral methods to the computation of the one-loop quantum correction to the energy per unit length of a AbrikosovNielsen-Olesen vortex. Because of the singular (topological) structure of this configuration, identifying the ultraviolet divergences that must be renormalized is nontrivial. In particular, the standard approach of equating elements of the Born series for the scattering data with (dimensionally) regularized Feynman diagrams fails because neither is well defined. This problem is confined to the coupling of the vector potential to the scalar (Higgs) quantum fluctuations, which we have therefore separated out in this study. As a first step, we considered a regular vector potential, and used the standard formulation of the spectral methods to verify that we then obtain the expected divergences. In doing so, we have identified a constant that must be subtracted from the angular momentum sum of the logarithm of the Jost function to maintain its analytic properties. This constant corresponds to the quadratic divergence of the loop diagrams with two insertions of the vector potential, which only cancels in a gauge invariant regularization. Hence this subtraction merely enforces gauge invariance. Since the quantum correction to the energy is an integral that contains the derivative of that sum, any constant may be subtracted without changing the result; we then integrate by parts for calculational convenience. We have then verified that no subleading logarithmic divergence is left over from these diagrams after subtraction of this constant other than the one eliminated by the standard wave-function renormalization of the gauge field. For nonsingular backgrounds, the behavior of the Jost function corresponding to this divergence sets in at relatively small momenta, for which the angular momentum sum converges reasonably fast. This situation changes dramatically for the singular background of the vortex. Not only is the onset of the correct asymptotic behavior shifted to larger momenta, depending on the strength of the scalar potential, but without proper extrapolation it may emerge from the sum over partial waves only when the sum is extended to extremely large values for which, unfortunately, the numerical calculation becomes unstable. For the generic example that we have studied exhaustively, no finite truncation of the angular momentum sum produced the correct behavior and an extrapolation was unavoidable. Furthermore, large angular momenta were required to obtain an accurate extrapolation. It may well be that the numerical effect is small when not matching the correct large momentum behavior and we could instead omit that part of the momentum integral when computing the vacuum polarization energy. Even if so, that can only be justified a posteriori and matching the asymptotics is essential for consistently renormalizing the quantum energy.

With these technical obstacles solved, the next step is to consider the full Higgs-gauge boson model that also treats the gauge fields quantum mechanically. For the transverse gauge modes there will be a scalar potential similar to $V_{H}$ and a coupling to the scalar quantum fluctuations by a vertex that connects to the vector potential profile. The temporal and longitudinal modes will only couple to the scalar potential. In three space dimensions, a particular 
gauge can be chosen such that the contributions to the quantum energy from the temporal and longitudinal modes cancel against those from the ghost fields needed for gauge fixing [6,20], while in two space dimensions this cancellation does not occur and these modes must be explicitly included. Of course, the full model calculation will also require physical on-shell renormalization conditions. Their implementation requires the momentum space analysis of Feynman diagrams beyond the ultraviolet divergences. We have seen how to treat obstacles that in this context arise from the Fourier transform of the singular vortex background by expressing such Feynman diagrams as the Fourier transform of the elements of the (regular) field strength tensor. This will allow us to compute the VPE of a superconducting vortex [2].

Ultimately we will be able to compare the quantum corrections to classical configurations in different topological sectors by replacing $\varphi \rightarrow n \varphi$ and $f_{G} \rightarrow n f_{G}$ with integer $n$ in Eq. (2). This calculation will provide further insight into the Skyrme model picture for nuclei, which estimates the nuclei binding energies as differences of classical energies in sectors whose topological charge equals the number of nucleons and energies due to canonical quantization of rotational modes [21]. With respect to the $\hbar$ counting, these are the leading and next-to-next-to-leading order contribution, while the next-to-leading order, the vacuum polarization energy, is omitted. It does not contribute to the mass differences within a given topological sector, but may alter the picture when different sectors are compared.

It will also be feasible to apply the proposed method to supersymmetric extension of the vortex model because the techniques to include fermions have already been established [22]. There are (mainly analytical) results [20,23] based on mode number counting. However, such arguments can require more thorough investigation when renormalization is needed [24].

\section{ACKNOWLEDGMENTS}

N. G. is supported in part by the National Science Foundation (NSF) through Grant No. PHY-1820700. H.W. is supported in part by the National Research Foundation of South Africa (NRF) by Grant No. 109497.
[1] N. Graham, M. Quandt, and H. Weigel, Lect. Notes Phys. 777, 1 (2009).

[2] A. A. Abrikosov, Zh. Eksp. Teor. Fiz. 32, 1442 (1957) [Sov. Phys. JETP 5, 1174 (1957)], http://www.jetp.ac.ru/cgi-bin/ e/index/e/5/6/p1174?a=list.

[3] H. B. Nielsen and P. Olesen, Nucl. Phys. B61, 45 (1973).

[4] N. Graham and H. Weigel (to be published).

[5] J. Baacke, Phys. Rev. D 78, 065039 (2008).

[6] J. Baacke and N. Kevlishvili, Phys. Rev. D 78, 085008 (2008); 82, 129905(E) (2010).

[7] P. Pasipoularides, Phys. Rev. D 64, 105011 (2001).

[8] A. Alonso Izquierdo, W. Garcia Fuertes, M. de la Torre Mayado, and J. Mateos Guilarte, Phys. Rev. D 70, 061702 (2004).

[9] J. Mateos Guilarte, A. Alonso-Izquierdo, W. Garcia Fuertes, M. de la Torre Mayado, and M. J. Senosiain, Proc. Sci., ISFTG2009 (2009) 013 [arXiv:0909.2107].

[10] A. Alonso-Izquierdo, J. Mateos Guilarte, and M. de la Torre Mayado, Phys. Rev. D 94, 045008 (2016).

[11] Y. Ferreirós and A. González-Arroyo, Phys. Rev. D 90, 025004 (2014).

[12] H. J. de Vega and F. A. Schaposnik, Phys. Rev. D 14, 1100 (1976).
[13] R. G. Newton, Scattering Theory of Waves and Particles (Springer, New York, 1982), Chap. 12.

[14] N. Graham, R. L. Jaffe, V. Khemani, M. Quandt, M. Scandurra, and H. Weigel, Nucl. Phys. B645, 49 (2002).

[15] M. Bordag and K. Kirsten, Phys. Rev. D 53, 5753 (1996).

[16] N. Graham, R. L. Jaffe, M. Quandt, and H. Weigel, Phys. Rev. Lett. 87, 131601 (2001).

[17] N. Irges and F. Koutroulis, Nucl. Phys. B924 (2017) 178; 938, 957(E) (2019).

[18] W.H. Press et al., Numerical Recipes in Fortran (Cambridge University Press, Cambridge, England, 1996).

[19] N. Graham, M. Quandt, and H. Weigel, Phys. Rev. D 84, 025017 (2011).

[20] B. H. Lee and H. Min, Phys. Rev. D 51, 4458 (1995).

[21] O. V. Manko, N. S. Manton, and S. W. Wood, Phys. Rev. C 76, 055203 (2007).

[22] N. Graham, V. Khemani, M. Quandt, O. Schröder, and H. Weigel, Nucl. Phys. B707, 233 (2005).

[23] J. R. Schmidt, Phys. Rev. D 46, 1839 (1992).

[24] N. Graham and R. L. Jaffe, Nucl. Phys. B544, 432 (1999). 